\title{
Oridonin induces apoptosis and senescence by increasing hydrogen peroxide and glutathione depletion in colorectal cancer cells
}

\author{
FENG-HOU GAO $^{1 *}$, FENG LIU $^{1 *}$, WEI WEI ${ }^{2}$, LI-BIN LIU ${ }^{3}$, MANG-HUA XU ${ }^{1}$, \\ ZHU-YING GUO ${ }^{1}$, WEI LI ${ }^{3}$, BIN JIANG ${ }^{1}$ and YING-LI WU ${ }^{2}$

\begin{abstract}
${ }^{1}$ Number 3 People's Hospital, Affiliated to Shanghai Jiao-Tong University School of Medicine (SJTU-SM), Shanghai 201900;
${ }^{2}$ Department of Pathophysiology and Chemical Biology, Division of Shanghai Universities E-Institutes, Key Laboratory of Cell Differentiation and Apoptosis of National Ministry of Education, Shanghai Jiao-Tong University

School of Medicine (SJTU-SM), Shanghai 200025; ${ }^{3}$ Zhejiang Provincial Key Laboratory of Medical

Genetics, School of Life Sciences, Wenzhou Medical College, Wenzhou, Zhejiang, P.R. China
\end{abstract}

Received November 14, 2011; Accepted December 22, 2011

DOI: $10.3892 / \mathrm{ijmm} .2012 .895$

\begin{abstract}
We recently demonstrated that oridonin could induce apoptosis and senescence of colon cancer cells in vitro and in vivo. However, the underlying mechanism remains unknown. In this study, the involvement of reactive oxygen species in oridonin-induced cell death and senescence was investigated in colon adenocarcinoma-derived SW1116 cells. Oridonin increased intracellular hydrogen peroxide levels and reduced the glutathione content in a dose-dependent manner. $\mathrm{N}$-acetylcysteine, a reactive oxygen species scavenger, not only blocked the oridonin-induced increase in hydrogen peroxide and glutathione depletion, but also blocked apoptosis and senescence induced by oridonin, as evidenced by the decrease in Annexin V and senescence-associated $\beta$-galactosidasepositive cells and the inhibition of oridonin-induced upregulation of p53 and p16 and downregulation of c-Myc. Moreover, exogenous catalase could inhibit the increase in hydrogen peroxide and apoptosis induced by oridonin, but not the glutathione depletion and senescence. Furthermore, thioredoxin reductase (TrxR) activity was reduced by oridonin in vitro and in cells, which may cause the increase in hydrogen peroxide. In conclusion, the increase in hydrogen peroxide and glutathione depletion account for oridonin-induced apoptosis and senescence in colorectal cancer cells, and TrxR inhibition
\end{abstract}

Correspondence to: Dr Ying-Li Wu, Department of Pathophysiology and Chemical Biology, Division of Shanghai Universities E-Institutes, Key Laboratory of Cell Differentiation and Apoptosis of National Ministry of Education, Shanghai Jiao-Tong University School of Medicine (SJTU-SM), Shanghai 200025, P.R. China

E-mail: wuyingliwu@163.com

*Contributed equally

Key words: oridonin, colorectal cancer, hydrogen peroxide, apoptosis, senescence is involved in this process. Given the importance of TrxR as a novel cancer target in colon cancer, oridonin would be a promising clinical candidate. The mechanism of oridonin-induced inhibition of TrxR warrants further investigation.

\section{Introduction}

Reactive oxygen species (ROS) include hydrogen peroxide, nitric oxide, superoxide anions, hydroxyl radicals and peroxynitrite. A growing body of evidence suggests that ROS within cells act as second messengers in the regulation of many important cellular events, including transcription factor activation, gene expression and cellular proliferation, differentiation and senescence $(1,2)$. An excessive production of ROS frequently leads to serious damage in cells, while mild increases in ROS can induce cellular senescence $(3,4)$. ROS can therefore function as antitumorigenic agents (5-7). Many anticancer therapies, such as the chemotherapeutic agent doxorubicin, etoposide and cisplatin, in addition to radiotherapy and photodynamic therapy, can lead to the generation of superoxide and oxygen radicals within carcinoma cells $(8,9)$. In this sense, the modulation of oxidative stress could be a target of anticancer therapies.

A variety of proteins function as ROS scavengers, such as superoxide dismutase, catalase, glutathione peroxidase, thioredoxin reductase (TrxR), ascorbate, glutathione. Among them, TrxR has been suggested as a new target for anticancer drug development because TrxR is overexpressed in colon, pancreas, lung and other cancers (10-12) and knockdown of TrxR could dramatically reduce tumor growth and metastasis in a lung carcinoma mouse model (13). Developing the novel TrxR targeting compound is promising in the treatment of cancer (14).

Oridonin is an ent-kaurane diterpenoid derived from Rabdosia rubescens (15). Studies have shown that oridonin induces apoptosis in a variety of cancer cells including cells from prostate cancer, breast cancer, non-small cell lung cancer, acute leukemia, glioblastoma multiforme and melanoma (16-19). In addition, oridonin can also inhibit cell cycle progression and enhance phagocytosis of apoptotic cells by macrophages (20). 
However, the mechanisms underlying the antitumor activity of oridonin are not fully understood. Interestingly, exposure of acute promyelocytic leukemia NB4 cells to oridonin resulted in a significant increase in ROS generation while the ROS scavenger, $\mathrm{N}$-acetylcysteine (NAC), completely protected NB4 cells from oridonin-induced apoptosis (16). These findings indicate that ROS signaling is involved in oridonin-induced apoptosis. Recently, we demonstrated that oridonin could induce potent growth inhibition, apoptosis, and senescence of colorectal cancer cells in vitro and in vivo (21). However, the exact mechanism of this process remains largely unknown.

In the present study, the role of ROS and thioredoxin reductase in oridonin-induced cell death and senescence in human colorectal cancer (SW1116) cells were investigated.

\section{Materials and methods}

Cell culture. The colorectal cancer cell line SW1116 was purchased from the Shanghai Institutes for Biological Sciences. The cells were maintained in a humidified room air containing $5 \% \mathrm{CO}_{2}$ at $37^{\circ} \mathrm{C}$ and cultured in DMEM medium (Gibco-BRL) supplemented with $10 \%$ fetal bovine serum and $1 \%$ penicillinstreptomycin (Gibco-BRL). Cells in the logarithmic phase of growth were used in all experiments.

Reagents. Oridonin (98\% purity) provided by Dr Tang Qingjiu (Shanghai Academy of Agricultural Sciences) was dissolved in DMSO (Sigma, St. Louis, MO) at a stock concentration of $10 \mathrm{mg} / \mathrm{ml}$ and stored at $-20^{\circ} \mathrm{C}$. The cell-permeable ROS scavenger NAC was obtained from Sigma and dissolved in sterile $\mathrm{H}_{2} \mathrm{O}$ to a stock concentration of $100 \mathrm{mM}$. Catalase was obtained from Sigma and dissolved in $50 \mathrm{mM}$ potassium phosphate buffer at $4,733 \mathrm{U} / \mathrm{ml}$. All stock solutions were wrapped in foil and maintained at $4^{\circ} \mathrm{C}$ or $-20^{\circ} \mathrm{C}$.

Detection and measurement of intracellular hydrogen peroxide and superoxide anion concentrations. Two oxidation-sensitive fluorescent probe dyes, 2',7'-dichlorodihydrofluorescein diacetate (DCF-DA, Invitrogen, Molecular Probes, Eugene, OR) and dihydroethidium(DHE, Invitrogen Molecular Probes), were used to measure the intracellular hydrogen peroxide and superoxide anion concentrations, respectively. DCF-DA is deacetylated intracellularly by nonspecific esterases and is further oxidized by cellular peroxides to the fluorescent compound 2',7'-dichlorofluorescein. DHE is a fluorogenic probe that detects superoxide anion radicals with high selectivity. DHE is cell-permeable and reacts with superoxide anions to form ethidium, which in turn intercalates deoxyribonucleic acid and exhibits a red fluorescence. Briefly, cells were treated with oridonin in the presence or absence of NAC or catalase for the indicated time periods. After washing with phosphate-buffered saline (PBS), cells were incubated with $20 \mu \mathrm{M}$ DCF-DA or $5 \mu \mathrm{M} \mathrm{DHE}$ at $37^{\circ} \mathrm{C}$ for $30 \mathrm{~min}$ according to the manufacturer's instructions. The fluorescence signals were detected by a FACStar flow cytometer (Beckman Coulter). For each sample, 5,000 or 10,000 events were collected. Hydrogen peroxide and superoxide anion levels were expressed in terms of mean fluorescence intensity.

Detection of intracellular glutathione (GSH). Cellular GSH levels were analyzed using 5-chloromethylfluorescein diacetate
(CMFDA, Invitrogen, Molecular Probes). Cytoplasmic esterases convert nonfluorescent CMFDA to fluorescent 5-chloromethylfluorescein, which can then react with the glutathione. CMFDA is a useful membrane-permeable dye for determining levels of intracellular glutathione (22-25). Briefly, cells were treated with oridonin in the presence or absence of ROS scavengers, or catalase for the indicated time periods. After washing with PBS, the cells were incubated with $5 \mu \mathrm{M} \mathrm{CMFDA}$ at $37^{\circ} \mathrm{C}$ for $30 \mathrm{~min}$ according to the manufacturer's instructions. CMF fluorescence was detected by the FACStar flow cytometer (Beckman Coulter). For each sample, 5,000 or 10,000 events were collected.

Annexin V/PI staining. Apoptosis was determined using Annexin V-fluorescein isothiocyanate (FITC) staining and PI labeling. Annexin V can identify the externalization of phosphatidylserine during apoptotic progression and therefore can detect cells in early apoptosis. Briefly, cells were treated with oridonin in the presence or absence of NAC or catalase for the indicated time periods. After washing twice with cold PBS, cells were resuspended in $500 \mu \mathrm{l}$ of binding buffer [10 mM HEPES/NaOH (pH 7.4), $140 \mathrm{mM} \mathrm{NaCl}, 2.5 \mathrm{mM} \mathrm{CaCl}_{2}$ ] at a concentration of $1 \times 10^{6}$ cells/ml. Next, $5 \mu$ of Annexin V-FITC (Pharmingen, San Diego, CA) and $10 \mu \mathrm{l}$ of $20 \mu \mathrm{g} / \mathrm{ml}$ PI were added to these cells, which were analyzed with a FACStar flow cytometer (Beckman Coulter). Viable cells were negative for both PI and Annexin V, apoptotic cells were positive for Annexin V and negative for PI, and late apoptotic dead cells displayed both high Annexin $\mathrm{V}$ and PI labeling. Non-viable cells that had undergone necrosis were positive for PI and negative for Annexin V.

Cell senescence assay. Senescence-associated $\beta$-galactosidase activity was determined with a senescence detection kit (Biovision, Mountain View, CA) using fixed cells (26). The development of a blue color in the cytoplasm was detected and photographed using a Nikon (Nikon Instruments Inc., Lewisville, TX) inverted microscope equipped with a color CCD camera. Four pictures were taken of each well. $\beta$-galactosidase-stained cells and unstained cells were counted and used to calculate a final average ratio of the number of stained to unstained cells in each well.

Western blot analysis. Cells were incubated with oridonin and/ or NAC for the indicated time periods. Cells were then washed in PBS and suspended in five volumes of lysis buffer [20 mM HEPES (pH 7.9), 20\% glycerol, $200 \mathrm{mM} \mathrm{KCl,} 0.5 \mathrm{mM}$ EDTA, $0.5 \%$ NP-40, $0.5 \mathrm{mM}$ DTT, $1 \%$ protease inhibitor cocktail (Sigma)]. The lysates were then collected and stored at $-20^{\circ} \mathrm{C}$. Protein concentrations in the supernatants were determined by the Bradford method. Supernatant samples containing $20 \mu \mathrm{g}$ of total protein were resolved on 10-15\% SDS-PAGE gels and transferred onto Immobilon-P PVDF membranes (Millipore, MA) by electroblotting; membranes were then probed with antiPARP, p16, c-Myc and p53 (Santa Cruz Biotechnology, Inc., Santa Cruz, CA) primary antibodies and subsequently with horseradish peroxidase-conjugated secondary antibodies. The membrane blots were developed using the ECL kit (Amersham, Arlington Heights, IL).

Determination of TrxR activity by the dithionitrobenzene reduction assay. All experiments were performed in 96-well 
A
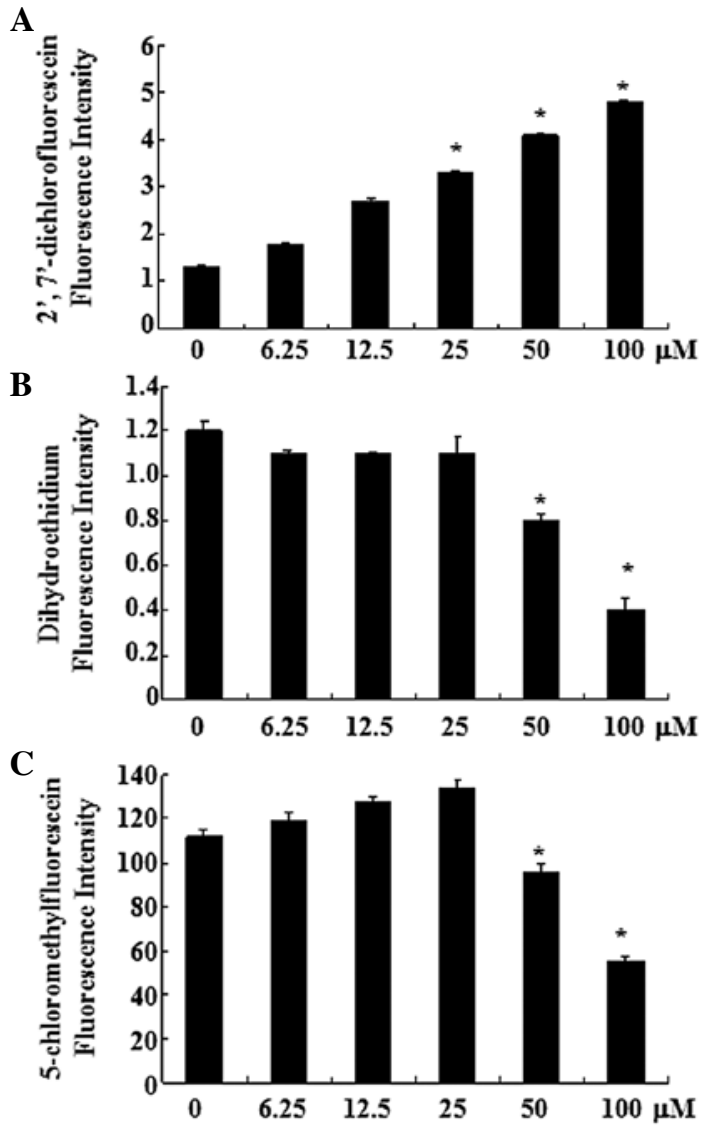

Figure 1. Effects of oridonin $(0,6.25,12.5,25,50$ or $100 \mu \mathrm{m})$ on intracellular hydrogen peroxide, superoxide anion and glutathione production in SW1116 cells. SW1116 cells were treated with the indicated concentrations of oridonin for $2 \mathrm{~h}$. Intracellular levels of (A) hydrogen peroxide, (B) superoxide anions, and (C) glutathione production were determined by Flow Cytometry. The graphs show the mean fluorescence levels of (A) DCF, (B) DHE and (C) CMF. Each experiment was repeated three times. ${ }^{*} \mathrm{P}<0.05$ when compared with the control group from three independent experiments.

plates. Recombinant active rat $\operatorname{TrxR}(0.05$ units) was incubated with various concentrations of oridonin for $60 \mathrm{~min}$. TrxR activity was assayed by the dithionitrobenzene method in a solution containing $50 \mathrm{mM}$ Tris- $\mathrm{HCl}(\mathrm{pH}$ 7.5), $200 \mu \mathrm{M}$ NADPH, $5 \mathrm{mM}$ DTNB, and $1 \mathrm{mM}$ EDTA. The absorbance at $412 \mathrm{~nm}$ was measured with a Synergy H4 Hybrid Microplate Reader (Bio-Tek, Winooski, VT). A blank reading without TrxR was subtracted from every sample. TrxR enzyme activity was calculated as a percentage of the activity of the DMSO vehicletreated sample.

Determination of TrxR activity in cell lysates. The activity of TrxR in cell lysates was measured using the TrxR assay kit (Cayman Chemical, Ann Arbor, MI) according to the manufacturer's instructions. Briefly, cells were collected by scraping and were homogenized on ice in cold buffer $(50 \mathrm{mM}$ potassium phosphate and 1 mM EDTA, $\mathrm{pH}$ 7.4). The protein concentration in the supernatant of the lysate was determined using the Bradford method. Protein samples, NADPH, and DTNB were added to the assay buffer in 96-well plates and gently mixed. The absorbance at $412 \mathrm{~nm}$ was measured with a Synergy H4 Hybrid Microplate Reader. A blank reading without protein was subtracted from every sample. TrxR enzyme activity was calculated as a percentage of the activity of the DMSO vehicletreated control sample.

Statistical analysis. The Student's t-test was used to evaluate the differences between two different groups. A P-value $<0.05$ was considered statistically significant.

\section{Results}

Oridonin increases hydrogen peroxide and decreases superoxide anion and glutathione levels in SW1116 cells. First, the production of intracellular hydrogen peroxide was assessed in oridonin-treated SW1116 cells using the DCF-DA fluorescence dye. As shown in Fig. 1A, oridonin increased the intracellular hydrogen peroxide level in a dose-dependent manner. Treatment with oridonin at $6.25 \mu \mathrm{M}$ for $2 \mathrm{~h}$ increased the intracellular hydrogen peroxide levels, compared with those in the untreated control cells. The intracellular superoxide anion level in oridonin-treated SW1116 cells was assessed using the DHE fluorescence dye. Red fluorescence derived from DHE, which reflects superoxide anion accumulation, decreased significantly in SW1116 cells treated with 50 and $100 \mu \mathrm{M}$ oridonin, compared with the untreated control cells (Fig. 1B).

As an important component of the antioxidant system, cellular GSH has been shown to be crucial for the regulation of cell proliferation, cell cycle progression, apoptosis and senescence. We therefore analyzed the changes of GSH level in oridonin-treated SW1116 cells by using the CMF fluorescence probe. Oridonin at 50 or $100 \mu \mathrm{M}$ significantly decreased the level of intracellular GSH content at $2 \mathrm{~h}$ (Fig. 1C), indicating the depletion of intracellular GSH content of SW1116 cells. The decrease of CMF fluorescence was observed within $120 \mathrm{~min}$ at the exposure to oridonin (50 and $100 \mu \mathrm{M})$ (Fig. 1C). However, at a lower concentration $(6.25-25 \mu \mathrm{M})$, a slight increase of the CMF fluorescence was detected at $2 \mathrm{~h}$.

The ROS scavenger NAC protects SW1116 cells from oridonininduced apoptosis or senescence. To determine the role of ROS production in oridonin-induced apoptosis and senescence, SW1116 cells were treated with oridonin in the presence or absence of NAC, a well known ROS scavenger, for $2 \mathrm{~h}$. As expected, NAC fully reversed the oridonin-induced increase in hydrogen peroxide as well as the decrease in superoxide anion and GSH levels (Fig. 2A-C). Next, effects of NAC on oridonin-induced cell death and senescence in SW1116 cells were also examined. As shown in Fig. 2D and E, co-treatment with NAC and oridonin completely protected the cells from oridonin-induced cell death and senescence.

NAC abrogates oridonin-induced changes in poly(ADPribose) polymerase (PARP), p16, p53 and c-Myc in SW1116 cells. Oridonin-induced apoptosis and senescence were related to the upregulation of p53 and p16 and downregulation of c-Myc (21). Because NAC can inhibit oridonin-induced apoptosis and senescence, the effect of NAC on the expression of these proteins was examined. As shown in Fig. 3A, the application of oridonin at $100 \mu \mathrm{M}$ mainly led to apoptosis in SW1116 cells, as indicated by the degradation of PARP, which is a major substrate for caspases and a marker for apoptosis (27). Co-treatment with NAC inhibited the degradation of PARP. At 


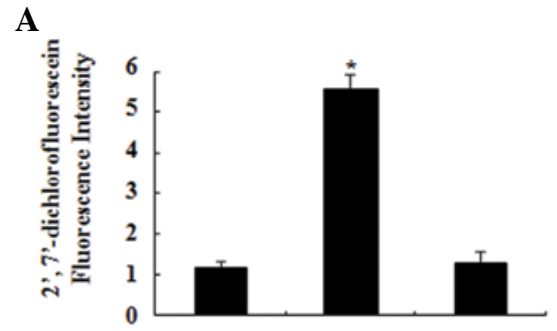

B

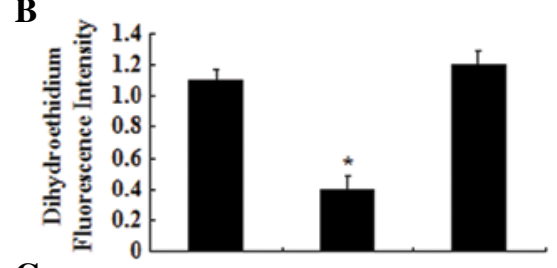

C

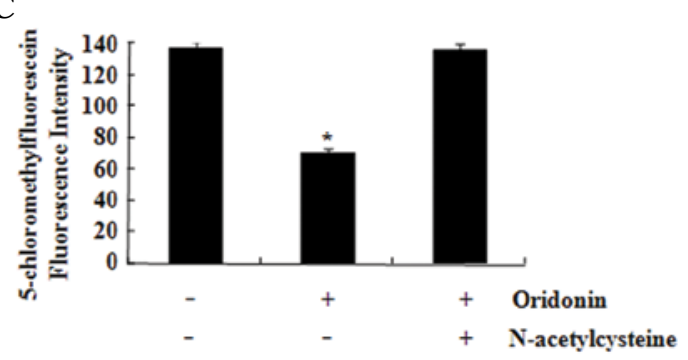

$\mathbf{D}$

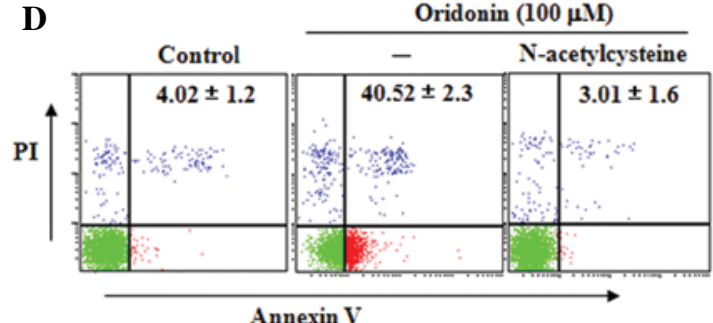

$\mathbf{E}$

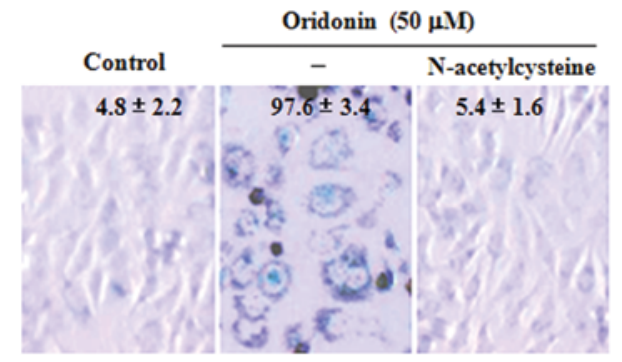

Figure 2. Effects of a ROS scavenger on intracellular ROS and GSH production, oridonin-induced apoptosis and senescence in oridonin-treated SW1116 cells SW1116 cells were treated with the ROS scavenger NAC and/or oridonin for $2 \mathrm{~h}$. Intracellular (A) hydrogen peroxide, (B) superoxide anion and (C) GSH levels were determined by FACS. The graphs show the mean fluorescence levels of (A) DCF, (B) DHE, and (C) CMF. (D-E) SW1116 cells were treated with NAC and/or oridonin for 12 or 48 h. (D) Annexin-positive cells were measured by flow cytometry. (E) Senescent cells were quantified by senescence-associated $\beta$-galactosidase activity analysis $(\mathrm{x} 100)$. Each experiment was repeated three times. " $\mathrm{P}<0.05$ when compared with the cells untreated or treated with oridonin plus NAC from three independent experiments.

A

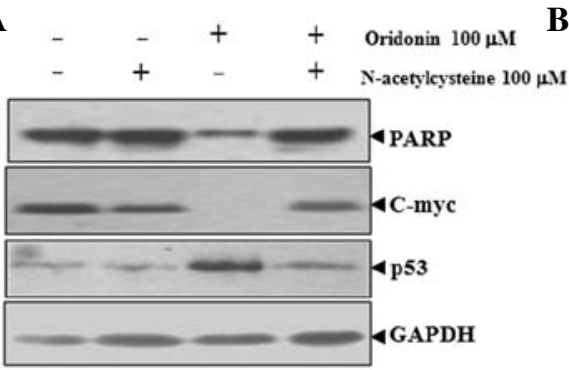

B

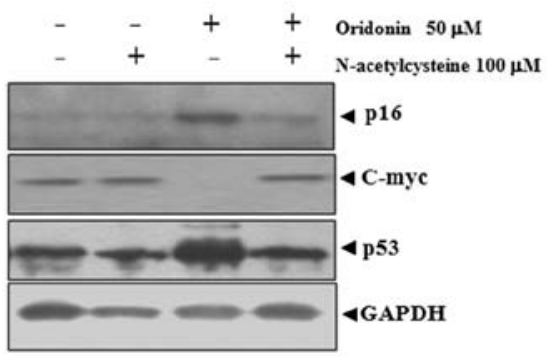

Figure 3. Effects of N-acetylcysteine on oridonin-induced apoptotic and senescence-related proteins in SW1116 cells. SW1116 cells were treated with NAC and/or oridonin for (A) $12 \mathrm{~h}(100 \mu \mathrm{M})$ and (B) $48 \mathrm{~h}(50 \mu \mathrm{M})$. Aliquots of protein extracts $(40 \mu \mathrm{g})$ were immunoblotted with the indicated antibodies.

$50 \mu \mathrm{M}$, oridonin mainly induced senescence in SW1116 cells, as reflected by the induction of p16, a hallmark of both replicative and accelerated senescence (28). In this case, co-treatment with NAC inhibited the induction of p16 (Fig. 3B).

The inactivation of c-Myc and the activation of p53 are important for both apoptosis and senescence (29-32). NAC co-treatment abrogated oridonin-induced downregualtion of c-Myc and upregulation of $\mathrm{p} 53$ in parallel with the inhibition of apoptosis and senescence (Fig. 3). These results indicated that c-Myc and p53 are involved in oridonin-induced senescence and apoptosis.

Effects of catalase on ROS production, apoptosis and senescence in oridonin-treated SW1116 cells. To further determine the role of hydrogen peroxide in oridonin-induced apoptosis and senescence, SW1116 cells were treated with oridonin in the presence or absence of catalase $(30 \mathrm{U} / \mathrm{ml})$, a hydrogen peroxide-scavenging enzyme (33). Catalase partially reversed an oridonin-induced increase in hydrogen peroxide (Fig. 4A) but not the decrease of superoxide anion and GSH (Fig. 4B and C). Interestingly, catalase significantly decreased the percentage of Annexin V-positive cells (Fig. 4D) but not the senescence-associated $\beta$-galactosidase-positive cells induced by oridonin (Fig. 4E).

Oridonin inhibits $\operatorname{Tr} x R$ activity. TrxR, an oxidoreductase that reduces oxidized proteins, represents an important valid target for cancer therapy. Because TrxR is easily targeted by electrophilic compounds and oridonin is a Michael acceptor with electrophilic propensity, we assumed that oridonin may 
A
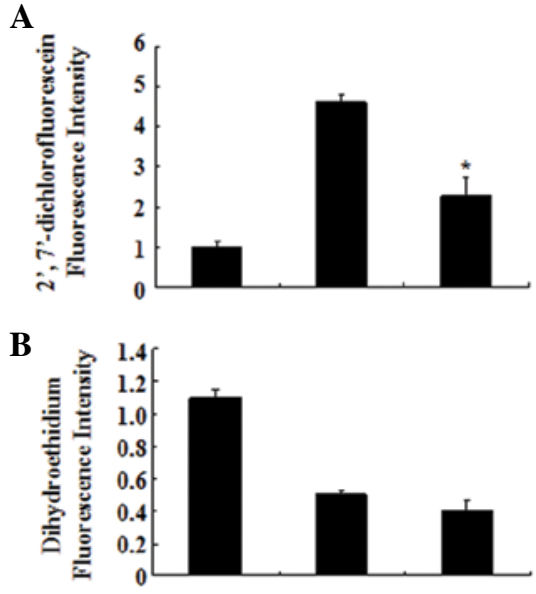

C

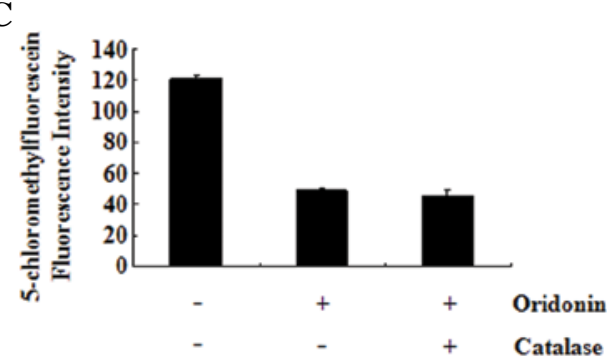

D

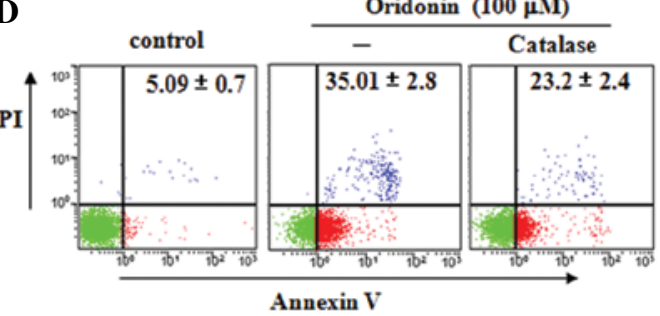

$\mathbf{E}$

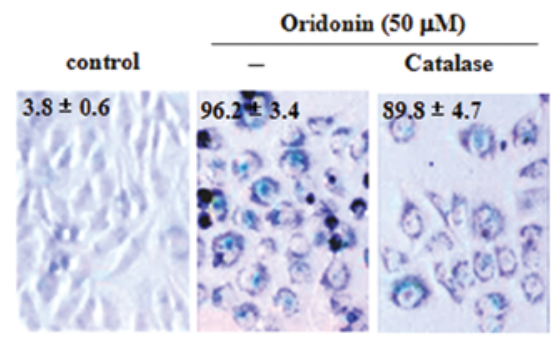

Figure 4. Effects of exogenous catalase on intracellular ROS production, glutathione depletion, oridonin-induced apoptosis and senescence in oridonin-treated SW1116 cells. SW1116 cells were treated with catalase and/or oridonin for $2 \mathrm{~h}$. Intracellular (A) hydrogen peroxide, (B) superoxide anion, and (C) GSH levels were determined by FACS, respectively. The graphs show the mean fluorescence levels of (A) DCF, (B) DHE and (C) CMF, respectively. Cells were treated with catalase and/or oridonin (50 $\mu \mathrm{M}$ for $48 \mathrm{~h}$ or $100 \mu \mathrm{M}$ for $12 \mathrm{~h}$ ). (D) Annexin V-positive cells were measured with FACS. (E) Senescent cells were determined by senescence-associated $\beta$-galactosidase activity analysis $(\mathrm{x} 100) .{ }^{*} \mathrm{P}<0.05$ compared with the cells treated with oridonin alone.

A

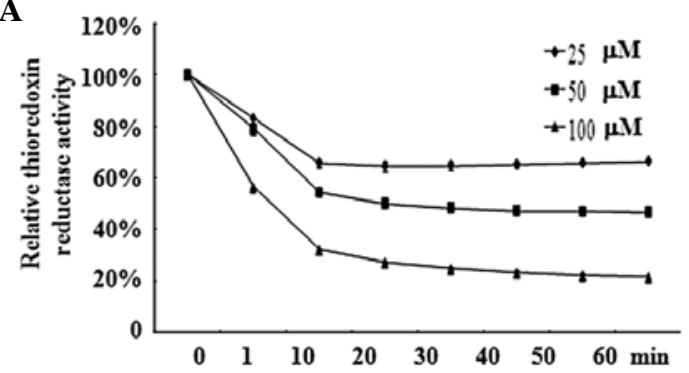

B

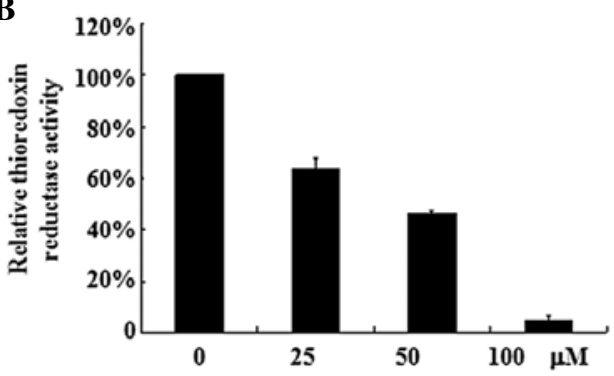

Figure 5. Inhibitory activity of oridonin on TrxR. (A) Recombinant rat TrxR ( 0.05 units) was incubated with various concentrations of oridonin for 1 h, and TrxR activity was measured by the dithionitrobenzene reduction assay. Error bars represent the standard deviations of duplicate experiments. (B) SW1116 cells were treated with different concentrations of oridonin for $2 \mathrm{~h}$, and TrxR activity was determined. Error bars represent the standard deviations of duplicate experiments.

directly inhibit TrxR activity, which causes an increase of hydrogen peroxide. As shown in Fig. 5A, oridonin caused a dose-dependent reduction of TrxR activity in vitro. Incubation of TrxR with $100 \mu \mathrm{M}$ oridonin led to an $80 \%$ decrease in TrxR activity compared with DMSO treatment. In line with this result, SW 1116 cells treated with oridonin at 50 or $100 \mu \mathrm{M}$ for $2 \mathrm{~h}$ exhibited a marked decrease in TrxR activity (Fig. 5B). These results indicate that $\operatorname{Trx} R$ is one of the targets for oridonin.

\section{Discussion}

We previously reported that oridonin induces potent growth inhibition, apoptosis, and senescence of colorectal cancer cells in vitro and in vivo (21). However, the underlying mechanism is largely unknown. In this study, we demonstrated that the antitumor activity of oridonin was attributed to its ROS increasing activity. Furthermore, TrxR inhibition is involved in this process.

ROS serve many cellular functions including actins as growth stimulants, second messengers, aging accelerants, cell death inducers and antibacterial agents. Based on our previous results and those of others $(16,34)$, we hypothesized that oridonin may induce apoptosis and senescence in colon cancer cells via increasing ROS. Although it is currently not possible to precisely quantify the amount of subcellular ROS, tools to detect several kinds of ROS in the cells are available. With these chemical probes, we found that exposure to 
oridonin led to the increase of hydrogen peroxide and decrease of superoxide anions and GSH in SW116 cells and that the effects of oridonin on ROS level were dose-dependent. In the cellular environment, hydrogen peroxide can be derived from superoxide anions; this derivation can either occur spontaneously or can be catalyzed by superoxide dismutase (35). Whether oridonin could enhance the conversion of superoxide anions to hydrogen peroxide or inhibit the production of superoxide anions is currently unknown.

Many studies have demonstrated that hydrogen peroxide plays an important role in chemotherapy-induced apoptosis and senescence in cells (6-9). At low or mild concentrations, ROS seem to protect the cells or induce cell senescence, while at high concentration, ROS can initiate cell death by damaging many biological molecules. To reveal the role of hydrogen peroxide in oridonin-induced apoptosis, NAC, an ROS scavenger, were used in combination with oridonin. NAC completely abrogated the oridonin-induced increases of hydrogen peroxide levels and decreases of superoxide anion levels. Accordingly, oridonin induced apoptosis and senescence and the relevant changes of p53, p16 and c-Myc were also abrogated. These data indicate that hydrogen peroxide plays a key role in oridonin-induced apoptosis and senescence. Interestingly, when the levels of hydrogen peroxide were partially decreased by catalase, only the apoptosis but not the senescence was significantly reversed, indicating that the concentration of hydrogen peroxide determined the cell fate. These data suggest that hydrogen peroxide may play a major role in oridonin-induced apoptosis and senescence, although we cannot rule out tht other ROS, such as nitric oxide, or hydroxyl radicals may also be involved.

The mammalian thioredoxin system, which consists of NADPH, thioredoxin and thioredoxin reductase, plays an important role in protecting cells from oxidative stressinduced apoptosis (36). As overexpression of TrxR has been reported in several human cancers and is associated with decreased survival and resistance to chemotherapy, TrxR has been proposed as a target for anticancer drug development. Considering the extensive involvement of TrxR in the redox regulations and its close link with cancer and that it is easily attacked by electrophilic compound, we investigated the effect of oridonin on TrxR activity. Interestingly, oridonin dosedependently inhibited the activity of TrxR in vitro and in cells. The trend was correlated to that of oridonin-induced hydrogen peroxide increase. Thus, oridonin may induce the accumulation of hydrogen peroxide by targeting TrxR.

In summary, we show here that hydrogen peroxide plays an important role in oridonin-induced apoptosis and senescence in colon cancer cells. We also provided evidence for the first time that oridonin could inhibit TrxR activity.

\section{Acknowledgements}

This study was supported by the National Basic Research Program of China (no. 2010CB912104), the National Natural Science Foundation of China (nos. 81070433, 91013008 and 81172322), the Science and Technology Committee of Baoshan District (08-E-13), and the Number 3 People's Hospital affiliated with the Shanghai Jiao-Tong University School of Medicine (syz07-04).

\section{References}

1. Gonzalez C, Sanz-Alfayate G, Agapito MT, Gomez-Nino A, Rocher A and Obeso A: Significance of ROS in oxygen sensing in cell systems with sensitivity to physiological hypoxia. Respir Physiol Neurobiol 132: 17-41, 2002.

2. Baran CP, Zeigler MM, Tridandapani S and Marsh CB: The role of ROS and RNS in regulating life and death of blood monocytes. Curr Pharm Des 10: 855-866, 2004.

3. Yamada J, Yoshimura S, Yamakawa H, et al: Cell permeable ROS scavengers, Tiron and Tempol, rescue PC12 cell death caused by pyrogallol or hypoxia/reoxygenation. Neurosci Res 45: 1-8, 2003.

4. Takahashi A, Ohtani N, Yamakoshi K, et al: Mitogenic signalling and the p16INK4a-Rb pathway cooperate to enforce irreversible cellular senescence. Nat Cell Biol 8: 1291-1297, 2006.

5. Mena S, Ortega A and Estrela JM: Oxidative stress in environmental-induced carcinogenesis. Mutat Res 674: 36-44, 2009.

6. Shaw AT, Winslow MM, Magendantz M, et al: Selective killing of K-ras mutant cancer cells by small molecule inducers of oxidative stress. Proc Natl Acad Sci USA 108: 8773-8778, 2011.

7. Raj L, Ide T, Gurkar AU, et al: Selective killing of cancer cells by a small molecule targeting the stress response to ROS. Nature 475: 231-234, 2011.

8. Engel RH and Evens AM: Oxidative stress and apoptosis: a new treatment paradigm in cancer. Front Biosci 11: 300-312, 2006.

9. Storz P: Reactive oxygen species in tumor progression. Front Biosci 10: 1881-1896, 2005.

10. Sun Y and Rigas B: The thioredoxin system mediates redoxinduced cell death in human colon cancer cells: implications for the mechanism of action of anticancer agents. Cancer Res 68: 8269-8277, 2008.

11. Han H, Bearss DJ, Browne LW, Calaluce R, Nagle RB and Von Hoff DD: Identification of differentially expressed genes in pancreatic cancer cells using cDNA microarray. Cancer Res 62: 2890-2896, 2002.

12. Soini Y, Kahlos K, Napankangas U, et al: Widespread expression of thioredoxin and thioredoxin reductase in non-small cell lung carcinoma. Clin Cancer Res 7: 1750-1757, 2001.

13. Yoo MH, Xu XM, Carlson BA, Gladyshev VN and Hatfield DL: Thioredoxin reductase 1 deficiency reverses tumor phenotype and tumorigenicity of lung carcinoma cells. J Biol Chem 281: 13005-13008, 2006.

14. Mukherjee A and Martin SG: The thioredoxin system: a key target in tumour and endothelial cells. Br J Radiol 81: S57-S68, 2008.

15. Ren KK, Wang HZ, Xie LP, et al: The effects of oridonin on cell growth, cell cycle, cell migration and differentiation in melanoma cells. J Ethnopharmacol 103: 176-180, 2006.

16. Gao F, Tang Q, Yang P, Fang Y, Li W and Wu Y: Apoptosis inducing and differentiation enhancement effect of oridonin on the all-trans-retinoic acid-sensitive and -resistant acute promyelocytic leukemia cells. Int J Lab Hematol 32: e114-e122, 2010.

17. Zhou GB, Kang H, Wang L, et al: Oridonin, a diterpenoid extracted from medicinal herbs, targets AML1-ETO fusion protein and shows potent antitumor activity with low adverse effects on $t(8 ; 21)$ leukemia in vitro and in vivo. Blood 109: 3441-3450, 2007.

18. Ikezoe T, Chen SS, Tong XJ, Heber D, Taguchi H and Koeffler HP: Oridonin induces growth inhibition and apoptosis of a variety of human cancer cells. Int J Oncol 23: 1187-1193, 2003.

19. Marks LS, DiPaola RS, Nelson P, et al: PC-SPES: herbal formulation for prostate cancer. Urology 60: 369-377, 2002.

20. Hsieh TC, Wijeratne EK, Liang JY, Gunatilaka AL and Wu JM: Differential control of growth, cell cycle progression, and expression of NF-kappaB in human breast cancer cells MCF-7, MCF-10A, and MDA-MB-231 by ponicidin and oridonin, diterpenoids from the chinese herb Rabdosia rubescens. Biochem Biophys Res Commun 337: 224-231, 2005.

21. Gao $\mathrm{FH}, \mathrm{Hu} \mathrm{XH}, \mathrm{Li} \mathrm{W}$, et al: Oridonin induces apoptosis and senescence in colorectal cancer cells by increasing histone hyperacetylation and regulation of p16, p21, p27 and c-myc. BMC Cancer 10: 610, 2010.

22. Poot M,Kavanagh TJ, Kang HC, Haugland RPand Rabinovitch PS: Flow cytometric analysis of cell cycle-dependent changes in cell thiol level by combining a new laser dye with Hoechst 33342. Cytometry 12: 184-187, 1991

23. Macho A, Hirsch T, Marzo I, et al: Glutathione depletion is an early and calcium elevation is a late event of thymocyte apoptosis. J Immunol 158: 4612-4619, 1997. 
24. Hedley DW and Chow S: Evaluation of methods for measuring cellular glutathione content using flow cytometry. Cytometry 15: 349-358, 1994.

25. Papiez MA, Dybala M, Sowa-Kucma M, et al: Evaluation of oxidative status and depression-like responses in Brown Norway rats with acute myeloid leukemia. Prog Neuropsychopharmacol Biol Psychiatry 33: 596-604, 2009.

26. Schmitt CA: Senescence, apoptosis and therapy - cutting the lifelines of cancer. Nat Rev Cancer 3: 286-295, 2003.

27. Decker P, Isenberg D and Muller S: Inhibition of caspase-3-mediated poly(ADP-ribose) polymerase (PARP) apoptotic cleavage by human PARP autoantibodies and effect on cells undergoing apoptosis. J Biol Chem 275: 9043-9046, 2000.

28. Gewirtz DA, Holt SE and Elmore LW: Accelerated senescence: an emerging role in tumor cell response to chemotherapy and radiation. Biochem Pharmacol 76: 947-957, 2008.

29. Di X, Shiu RP, Newsham IF and Gewirtz DA: Apoptosis, autophagy, accelerated senescence and reactive oxygen in the response of human breast tumor cells to adriamycin. Biochem Pharmacol 77: 1139-1150, 2009.

30. Jones KR, Elmore LW, Jackson-Cook C, et al: p53-Dependent accelerated senescence induced by ionizing radiation in breast tumour cells. Int J Radiat Biol 81: 445-458, 2005.
31. Barrett JC, Annab LA, Alcorta D, Preston G, Vojta P and Yin Y: Cellular senescence and cancer. Cold Spring Harb Symp Quant Biol 59: 411-418, 1994.

32. Quick QA and Gewirtz DA: An accelerated senescence response to radiation in wild-type p53 glioblastoma multiforme cells. J Neurosurg 105: 111-118, 2006.

33. Sousa T, Pinho D, Morato M, et al: Role of superoxide and hydrogen peroxide in hypertension induced by an antagonist of adenosine receptors. Eur J Pharmacol 588: 267-276, 2008

34. Zhang YH, Wu YL, Tashiro SI, Onodera S and Ikejima T: Reactive oxygen species contribute to oridonin-induced apoptosis and autophagy in human cervical carcinoma HeLa cells. Acta Pharmacol Sin 32: 1266-1275, 2011.

35. Reth M: Hydrogen peroxide as second messenger in lymphocyte activation. Nat Immunol 3: 1129-1134, 2002.

36. Holmgren $\mathrm{A}$ and $\mathrm{Lu} \mathrm{J}$ : Thioredoxin and thioredoxin reductase: current research with special reference to human disease. Biochem Biophys Res Commun 396: 120-124, 2010. 